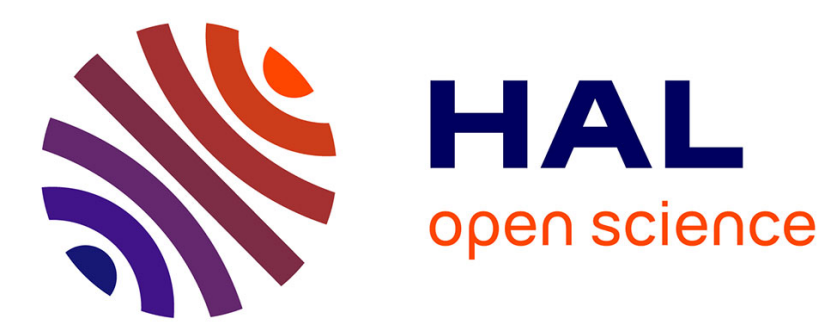

\title{
Eventual Consistency
}

Marc Shapiro, Bettina Kemme

\section{To cite this version:}

Marc Shapiro, Bettina Kemme. Eventual Consistency. Ling Liu; M. Tamer Özsu. Encyclopedia of Database Systems, Springer, pp.1-2, 2017, 978-1-4899-7993-3. 10.1007/978-1-4899-7993-3_1366-2 . inria-00444791v2

\section{HAL Id: inria-00444791 https://hal.inria.fr/inria-00444791v2}

Submitted on 26 Jun 2017

HAL is a multi-disciplinary open access archive for the deposit and dissemination of scientific research documents, whether they are published or not. The documents may come from teaching and research institutions in France or abroad, or from public or private research centers.
L'archive ouverte pluridisciplinaire HAL, est destinée au dépôt et à la diffusion de documents scientifiques de niveau recherche, publiés ou non, émanant des établissements d'enseignement et de recherche français ou étrangers, des laboratoires publics ou privés. 
Encylopedia of Database Systems, 2016 edition

\title{
Eventual Consistency
}

\author{
Marc Shapiro \\ Sorbonne-Universités-UPMC-LIP6 \& Inria Paris \\ Bettina Kemme \\ School of Computer Science, McGill University, Montreal, QC, Canada
}

2016

\section{Synonyms}

Lazy replication; Optimistic replication

\section{Definition}

In a replicated database, the consistency level defines whether and how the values of the replicas of a logical object may diverge in the presence of updates. Eventual consistency is the weakest consistency level that guarantees convergence. Informally, it requires that all replicas of an object will eventually reach the same final value, assuming that no new updates are submitted to the object.

\section{Formal Definition and Usage}

Eventual consistency is an important correctness criterion in systems with a lazy, updateanywhere strategy, also referred to as optimistic replication. Update operations can be submitted and executed on any node, and the propagation of updates occurs lazily after they are committed. Conflict resolution and reconciliation must ensure that all replicas (copies) of a logical object eventually converge to the same value. Different objects are considered independent. Especially in wide-area settings, also referred to as geo-replication, and mobile computing environments, eventual consistency is popular, as it allows individual replicas to serve client requests and provide a response before coordinating with other replicas. Conflict-free replicated data types (CRDTs) were invented to encapsulate and hide the complexity of managing eventual consistency. 
In a system where updates are continuously submitted, eventual consistency can be defined by a weak form of schedule equivalence [1]. A schedule $S_{n}^{x}$ describes the sequence of update operations that a node $n$ performs on its replica of object $x$. An element of the schedule of the form $w_{i}$ represents the execution of an update to object $x$, submitted by some user. $S_{n}^{x}$ contains an element of the form $\overline{w_{i}}$, if the update $w_{i}$ was received by $n$, but either not executed, or aborted due to conflict resolution.

Typically, two schedules are defined equivalent by restricting how the order of operations in the two schedules may differ. However, for eventual consistency, only the final convergence of object values matters. Thus, equivalence is defined by comparing the final state of the replicas. Two schedules are said state equivalent when, starting from the same initial state, they produce the same final state. For instance, (i) schedules $S=w_{1} w_{2}$ and $S_{0}=w_{2} w_{1}$ are state-equivalent if $w_{1}$ and $w_{2}$ commute; (ii) schedules $S=w_{1} w_{2}$ and $S^{\prime}=w_{2}$ are state-equivalent if $w_{2}$ overwrites the state of the object to a completely new value (e.g., $x:=2$ ). Eventual consistency of a replicated object $x$ is defined by the following conditions, which must hold at all times, at any node $n$ with a replica of $x$ [1]. It is assumed that all replicas have the same initial state:

- There is a prefix of the schedule $S_{n}^{x}$ that is state equivalent to a prefix of the schedule $S_{n}^{x^{\prime}}$ of any other node $n^{\prime}$ holding a replica of $x$. Such a prefix is called a committed prefix of $S_{n}^{x}$.

- The committed prefix of $S_{n}^{x}$ grows monotonically over time, i.e., the set of operations and their relative order remain unchanged.

- For every operation $w_{i}$ submitted by a user, either $w_{i}$ or $\overline{w_{i}}$ eventually appears in the committed prefix of $S_{n}^{x}$ (but not both and not more than once).

- An operation of the form $w_{i}$ in the committed prefix satisfies all its preconditions (e.g., the state of the object immediately before the execution of the operation fulfills certain conditions).

As an example, assume operation $w_{1}$ sets $x$ to 2 , and $w_{2}$ sets it to 5 . Operation $w_{1}$ is submitted and executed at node $n_{1}$, while $w_{2}$ is first executed at $n_{2}$. At this time, the local schedules are $S_{1}=w_{1}$ and $S_{2}=w_{2}$ and the committed prefix at both nodes is the empty schedule. Now $w_{1}$ is propagated to $n_{2}$, and $w_{2}$ is propagated to $n_{1}$. When $n_{1}$ receives $w_{2}$, it detects that $w_{1}$ and $w_{2}$ are concurrent and conflict. Say that conflict reconciliation prioritizes one of the operations, e.g., $w_{1}$. Then, $w_{2}$ is simply not executed and the new schedule is $S_{1}^{x}=w_{1} \overline{w_{2}}$. At $n_{2}$, when $w_{1}$ arrives, the conflict is also detected, $w_{2}$ is undone, $w_{1}$ is executed and the final schedule is $S_{2}=\overline{w_{2}} w_{1}$. At this time, $S_{1}$ and $S_{2}$ are themselves the committed prefixes. Note that further concurrent operations on $x$ might move the schedules further, but the extensions would still be tentative and only become committed once they are reconciled at all replicas.

\section{Cross-References}

- Consistency Models for Replicated Data 
- Optimistic Replication and Resolution

- Replicated Data Types

- WAN Replication

\section{Recommended Reading}

[1] Yasushi Saito and Marc Shapiro. Optimistic replication. ACM Computing Surveys, 37(1):42-81, March 2005. doi: 1057977.1057980. URL http://doi.acm.org/10.1145/ 1057977.1057980.

[2] Doug Terry. Replicated data consistency explained through baseball. Communications of the ACM, 56(12):82-89, December 2013. doi: 10.1145/2500500. URL http://doi. acm.org/10.1145/2500500.

[3] Werner Vogels. Eventually consistent. ACM Queue, 6(6):14-19, October 2008. doi: http://doi.acm.org/10.1145/1466443.x. 